\title{
Developing New Solutions for a Reconfigurable Microstrip Patch Antenna by Inverse Artificial Neural Networks
}

\author{
Ashrf Aoad ${ }^{1} \quad$ Murat Simsek ${ }^{2}$ \\ ${ }^{1}$ Department of Electrical and Electronics Engineering, Istanbul Sabahattin Zaim University, Turkey, \\ ashawad@hotmail.com \\ ${ }^{2}$ Department of Astronautics Engineering, Istanbul Technical University, Turkey, simsekmu@itu.edu.tr
}

\begin{abstract}
This paper presents the use of inverse artificial neural networks (ANNs) to develop and optimize a reconfigurable 5-fingers shaped microstrip patch antenna. New solutions are produced by using three accurate prior knowledge inverse ANNs with sufficient amount of training data where the frequency information is incorporated into the structure of ANNs. The proposed antenna can operate with four modes, which are controlled by two PIN diode switches with ON/OFF states, and it resonates at multiple frequencies between 2-7 GHz. The complexity of the input/output relationship is reduced by using prior knowledge. Three independent methods of incorporating knowledge in the second step of the training process with a multilayer perceptron (MLP) in the first step are demonstrated and their results are compared to EM simulation.
\end{abstract}

Keywords: artificial neural networks, reconfigurable microstrip antenna, prior knowledge input

\section{Introduction}

With the rapid development of wireless communication applications, especially in satellites, MIMO systems, radar and portable computers (Costantine et al., 2015). The choice of reconfigurable antennas comes in a large variety of different shapes and forms of the structure. Through change the structure of reconfigurable antennas different characteristics (desired operation) can be obtained (Jiajie and Anguo, 2018; Allayioti and Kelly, 2017). In addition, they have the nature and the capabilities of the reconfiguration mechanism (Aoad et al., 2014). In this application, only ON-ON state of the PIN diode switches is studied (Costantine et al., 2015; Aoad et al., 2014). In a previous study, the structure and results were different (Aoad et al., 2015).

ANN models have been selected for developing new solutions of the proposed antenna. They have the opportunity for modelling and optimizing non-linear relationships between multiple outputs and inputs (Huff and Bernhard, 2008). Therefore, ANNs can be used in the design, development and optimization of antennas (Aoad et al., 2014), integrated circuit-antenna modules and microwave circuits (Wang and Zhang, 1997). The accuracy depends on sufficient training data presented during the training process. In this application, training data is generated by CST-EM simulator.

In this study, two-steps of EM-ANN model is processed using MLP in the first step to model the geometrical dimensions (response) of the proposed antenna, followed by adding extra knowledge into neural networks (NNs) in the second step to correct the response that achieved from first step. Finally, the obtained from second step will be redesigned by EMsimulator to be the developed new solution.

\section{Reconfigurable Antenna Design}

The studied antenna is a reconfigurable 5-fingers shaped microstrip patch antenna (R5SMPA). This R5SMPA consists of three layers and feeding system at the center of the middle patch. The radiating conductors (first layer) consist of three strips with different dimensions. The parameter of $L_{1}(1.35 \mathrm{~cm})$ and $L_{2}(0.75 \mathrm{~cm})$ are mirrored to the other side where $L_{3}(1.05 \mathrm{~cm})$ is the middle strip. All strips linked by $W_{1}(3.3 \mathrm{~cm})$ where $W_{2}$ $(0.3 \mathrm{~cm})$ is the width of all strips. They are positioned on FR-4 dielectric board (second layer) with a thickness of $0.2 \mathrm{~cm}$ and ground plane (third layer) is printed on the back side of the dielectric (substrate). $W_{3}(0.15 \mathrm{~cm})$ is the unfilled space includes two PIN diodes $\left(D_{1}\right.$ and $\left.D_{2}\right)$ (ITTC, 2016). They are positioned to distribute the current paths on the microstrips depending on its bias state as shown in Figure 1. To realize the ON-ON state two resistors are used of the PIN diodes (ITTC, 2016). Each resistor has a resistance value of 5 Ohms.

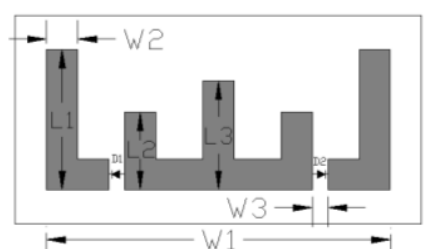

(a)

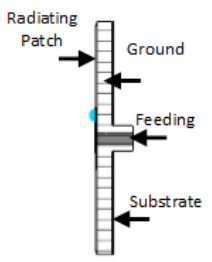

(b)
Figure 1. Reconfigurable antenna (a) Top view and (b) side view. 


\section{Inverse Artificial Neural Networks}

For testing the effectiveness of used methods, which were used in (Aoad et al., 2015). Same methods have been applied to the proposed antenna that has a different structure as shown in Figure 1. The proposed inverse ANNs consist of two-steps which is called knowledge based response correction (KBRC), followed by EM simulator to redesign the results of KBRC for obtaining new solutions of the R5SMPA. Input for KBRC model is only frequency sample points, while outputs are considered the new geometrical dimensions of the R5SMPA. It is important to notice that MLP response which is obtained from the first step of KBRC model is not corrected yet. However, SD, PKI-D and PKI models in the second step correct that response.

\subsection{Multilayer Perceptron (MLP)}

MLP (no extra knowledge) consists of three perceptron layers lined as an input layer, one or more hidden layers and finally an output layer (Zhang and Gupta, 2000) and is in the first step of KBRC model, corresponding to model $\mathrm{Y}$ and $\mathrm{X}$ variables respectively. The function of the input and the output vectors can be presented as $X=$ $f(Y)$. In this study, the input parameter is $Y_{f}=[f]^{T}\left(Y_{f}\right.$ presents 200 samples of S-parameters) and the predicted output is $X_{c}=\left[L_{1}, L_{2}, L_{3}\right]^{T}$.

\subsection{Source Difference Method (SD)}

The idea of SD (Simsek et al., 2010; Zhang and Gupta, 2000) is in combining two training data sets to be the target of the network. These data sets are the EM simulation outputs of $X_{f}=\left[L_{1}, L_{2}, L_{3}\right]^{T}$ which represents the fine data and the output response of MLP $\left(X_{c}\right)$ obtained from the first step. Thus, the input parameter of the SD is only the frequency samples $Y_{f}=$ $[f]^{T}$, the predicted output $X_{S D}=X_{C}+X_{M L P}$, while the target is $\Delta X_{S D}=X_{R L}-X_{C}$. SD is positioned in the second step of KBRC as shown in Figure 2. The function of the input and the output of the redesign case of EM-simulation is presented as

$$
Y_{f-S D}=f_{E M}\left(X_{S D}\right)
$$

where $Y_{f-S D}$ is the result obtained by the redesign of the predicted output $\left(X_{S D}\right)$ of the second step. $e_{S D}$ is the error measure computes the absolute difference between $Y_{f-S D}$ and $Y_{f}$ which can be calculated by

$$
e_{S D}=\left|Y_{f-S D}-Y_{f}\right|
$$

Equations (1) and (2) are same as a general principle in the next methods.

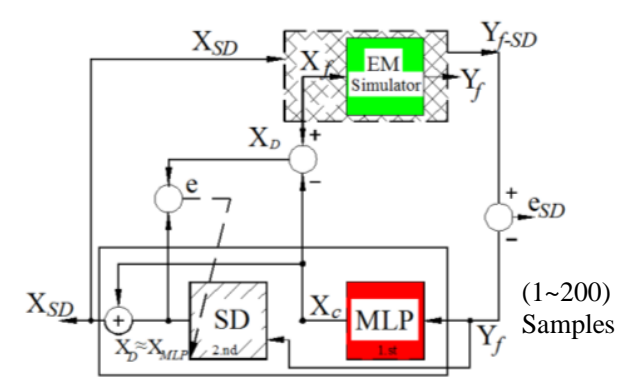

Figure 2. Two steps of KBRC model when SD is in 2nd step of processing.

\subsection{Prior Knowledge Input Method (PKI)}

In this method (Zhang and Gupta, 2000), the output response of MLP $\left(X_{C}\right)$ is used as input to PKI, in addition to the original input of $Y_{f}$. The target output is the fine output $\left(X_{f}\right)$. Therefore, the input/output mapping (in the first step) is between the output response of MLP $\left(X_{c}\right)$ and $\left(Y_{f}\right)$. Thus, the input parameter for PKI is $Y_{P K I}=\left[Y_{f}, X_{C}\right]^{T}$. PKI is positioned in the second step of KBRC as shown in Figure 3 .

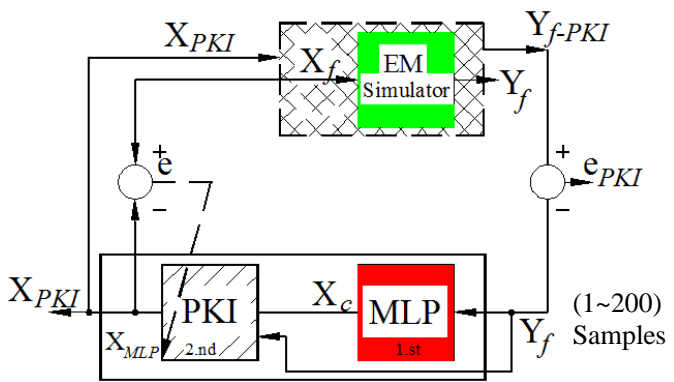

Figure 3. Two steps of KBRC model when PKI is in 2nd step of processing.

\subsection{Prior Knowledge Input with Different}

PKI-D (Aoad et al., 2014; Zhang \& Gupta, 2000) is developed to combine advantages of two knowledge based methods (PKI and SD) described previously. The prior knowledge obtained from the output response of $\operatorname{MLP}\left(X_{C}\right)$ is used with the input of the fine model $\left(Y_{f}\right)$ to be the input of PKI-D $\left(Y_{P K I-D}\right)$. Therefore, the input parameter is $Y_{P K I-D}=\left[Y_{f}, X_{C}\right]^{T}$, when the target output is $\Delta X_{P K I-D}=X_{f}-X_{C}$. PKI-D is positioned in the second step of KBRC as shown in Figure 4.

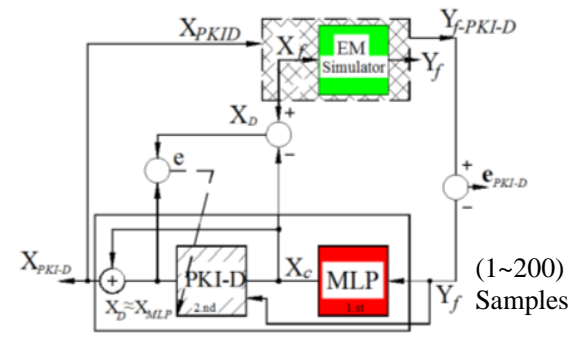

Figure 4. Two steps of KBRC model when PKI-D is in 2 nd step of processing. 


\section{Parameters of ANN Models}

Two data sets are initially proposed: 1) Training data and 2) extrapolation testing data sets. The training data generated by EM-simulator was 24800 samples for three geometric antenna parameters $\left(L_{i}^{k}, i=1,2,3\right.$ and $k=$ $5), k$ is the number of $L_{i}$ samples as shown below.

$$
N_{t r}=f_{s} \prod_{i=1}^{3}\left|L_{i}^{k}\right|
$$

where $N_{t r}$ is the number of training data samples and $f_{s}$ is the number of frequency samples which is equal to 200. The large amount of training data has been reduced to be 27 samples only (Bataineh and Marler, 2017). The reduction procedure depends on the selection of resonant frequency samples from the training data. The frequency sample points are 200 which are considered the input of the studied models and the outputs are three parameters which are the geometrical dimensions of the R5SMPA. In testing stage, two testing data sets are selected. The testing data sets are selected outside training data which are used to test the accuracy of the models for extrapolation (Simsek et al., 2010). The number of hidden layers is two for all methods. However, the number of neurons is (20-20) for MLP and (30-20) for knowledge based neural networks (KBNNs). Inverse ANN models are trained by using LevenbergMarguardt algorithm, with tangent-sigmoid transfer functions (TFs) in the hidden layers and a purely linear function in the output layer (Beale et al., 2013). The training parameters of the model are realized by adjusting the learning rate $(\eta)$ to 0.1 for MLP and 0.05 for KBNNs, the performance goal to 0.000001 for MLP and KBNNs and momentum coefficient $(\mu)$ to 0.2 for MLP and 0.1 for others. The regularization coefficient of the network is chosen as 0.2 .

\section{Results and Discussion}

The neural network models are repeatedly trained 50 times and new geometrical dimensions of R5SMPA are developed. The accuracy of the models is presented by the optimum resonant frequency and return loss of the S-parameter curves which are the results of the simulating the new geometrical parameters that obtained by inverse ANN models for extrapolation testing data. Figure 5 shows the result of R5SMPA without training any ANNs as explained in section 2 and shown in Figure 1(a), followed results are by using inverse ANNs.

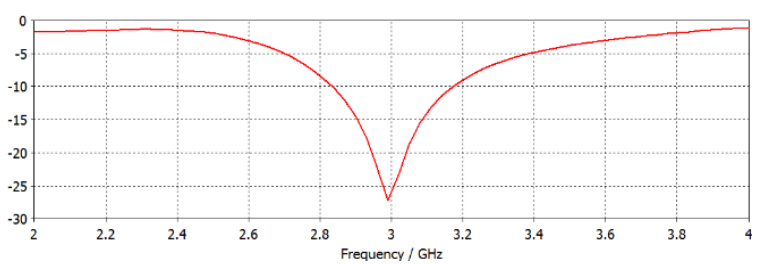

Figure 5. S-parameter for R5SMPA modeled by EM-Simulator, at $3 \mathrm{GHz}$.

Table 1. A Comparıson Between Results Obtained By Inverse ANNs.

\begin{tabular}{|c|c|c|c|c|c|}
\hline Parameters & Test & $M L P$ & $S D$ & $P K I$ & $P K I-D$ \\
\hline$L_{1}(\mathrm{~cm})$ & - & 1.4271 & 1.4738 & 1.3960 & 1.4377 \\
\hline$L_{2}(\mathrm{~cm})$ & - & 1.1115 & 1.0995 & 1.0818 & 1.1184 \\
\hline$L_{3}(\mathrm{~cm})$ & - & 0.8535 & 0.8496 & 0.8578 & 0.8340 \\
\hline$f_{o p}(\mathrm{GHz})$ & 2.44 & 2.71 & 2.68 & 2.74 & 2.71 \\
\hline$R L(\mathrm{~dB})$ & - & -26.89 & -32.00 & -25.91 & -25.73 \\
\hline
\end{tabular}

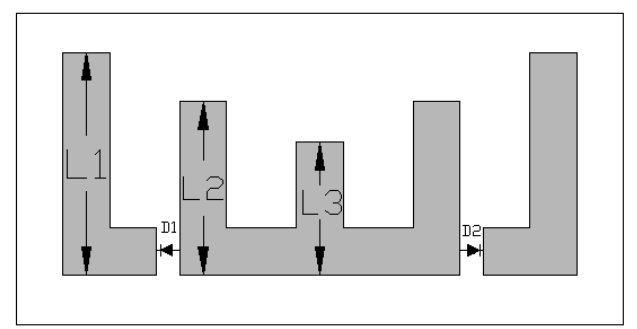

Figure 6. Top view of the developed solution for MLP only at $2.44 \mathrm{GHz}$.

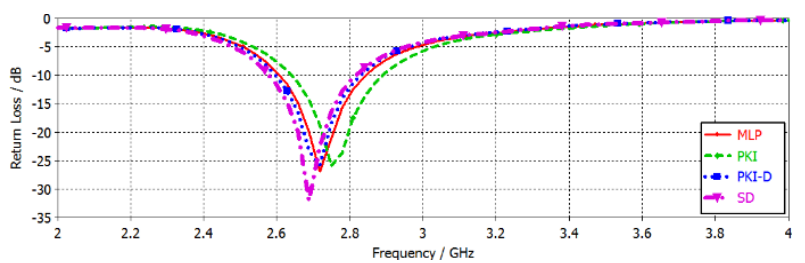

Figure 7. Comparison of S-parameters. The plots show results obtained by ANNs then designed by EM simulator, at $2.44 \mathrm{GHz}$.

Table 2. A Comparison Between Results Obtained by Inverse ANNs at $3.74 \mathrm{GHz}$ Extrapolation Testing Data.

\begin{tabular}{|c|c|c|c|c|c|}
\hline Parameters & Test & $M L P$ & $S D$ & $P K I$ & $P K I-D$ \\
\hline$L_{1}(\mathrm{~cm})$ & - & 0.5521 & 0.5445 & 0.5905 & 0.5466 \\
\hline$L_{2}(\mathrm{~cm})$ & - & 0.7800 & 0.7923 & 0.7745 & 0.7900 \\
\hline$L_{3}(\mathrm{~cm})$ & - & 0.9486 & 0.9618 & 0.9647 & 0.9689 \\
\hline$f_{o p}(\mathrm{GHz})$ & 3.74 & 3.77 & 3.74 & 3.77 & 3.77 \\
\hline$R L(\mathrm{~dB})$ & - & -21.05 & -21.11 & -22.47 & -22.05 \\
\hline
\end{tabular}

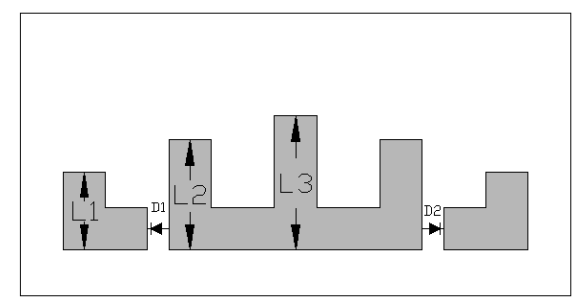

Figure 8. Top view of the developed solution for MLP only, at $3.74 \mathrm{GHz}$. 


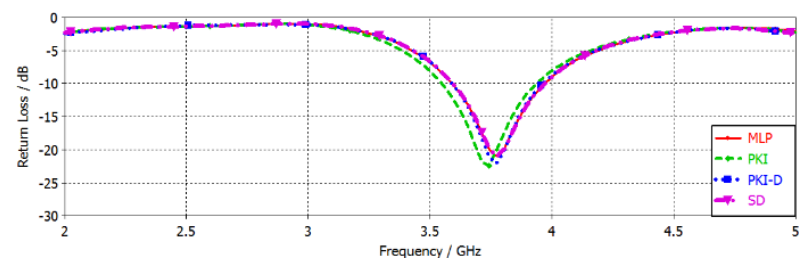

Figure 9. Comparison of S-parameters. The plots show results obtained by ANNs then designed by EM simulator, at $3.74 \mathrm{GHz}$.

Table 1 and Table 2 show the results of the redesigned physical parameters obtained by inverse ANN models, in addition to optimum frequencies and their return losses. The developed new solutions have new values and shapes for R5SMPA that differ from the original R5SMPA which is shown in Figure 1(a) and the result of Figure 5. Every solution obtained from ANNs operates successfully at a close resonant frequency band as shown in Figure 7 and Figure 9. Moreover, Figure 7 , and Figure 9 show that the bandwidths are around $0.32 \mathrm{GHz}$ and $0.41 \mathrm{GHz}$ respectively, at a target of return loss of $S_{11} \leq-10 \mathrm{~dB}$.

From the results presented above, it is noticed that ANN models are reliable and accurate models. The antenna can be reconfigured to obtain new results as well at different extrapolation testing data sets and new switching states such as ON-OFF or OFF-OFF. By comparing results with obtained in (Aoad et al., 2015). The difference was in resonating frequencies, their optimum points, bandwidths, number of input samples and speed of simulations.

\section{Conclusions}

The proposed inverse ANN methods have been applied for developing new solutions of the reconfigurable antenna. They consist of two steps of processing. MLP is in the first step, SD, PKI-D and PKI are in the second step to correct the response comes from MLP, then the results obtained by the second step redesigned by EM simulator. All methods is applicable to 2 hidden layers. Finally, after applying the proposed inverse ANN methods for developing new solutions of two different shaped reconfigurable antennas. The antenna designers can use same methods to design reconfigurable antennas to reach new solutions and high accuracy.

\section{References}

A. Aoad, M. Simsek and Z. Aydin. Design of a Reconfigurable 5-Fingers Shaped Microstrip Patch Antenna by Artificial Neural Networks. International Journal of Advanced Research in Computer Science and Software Engineering (IJARCSSE), 4 (10): 61-70, 2014.

Available via www.ijarcsse.com
A. Aoad, Z. Aydin and E. Korkmaz. Design of a Tri band 5Fingers Shaped Microstrip Patch Antenna with an Adjustable Resistor. Antenna Measurements \& Applications (CAMA), IEEE Conference. Antibes Juan-Les-Pins, 2014.

A. Aoad, M. Simsek and Z. Aydin. Development of Knowledge Based Response Correction for a Reconfigurable N-Shaped Microstrip Antenna Design. IEEE MTT-S International Conference. Ottawa, 2015. doi: 10.1109/NEMO.2015.7415078

F. Wang and Q. J. Zhang. Knowledge-Based Neural Models for Microwave Design. IEEE Transaction on Microwave Theory and Techniques, 45 (12): 2333-2343, 1997.

C. A. Balanis. Modern Antenna Handbook. John Wiley \& Sons, Inc. 369-395. 2008.

Information and Telecommunication Technology Center (ITTC). Retrieved $02 \quad 25, \quad 2016$, from http://www.ittc.ku.edu/ jstiles/622/handouts/old\%20hando uts/PIN\%20Diodes.pdf

J. Costantine, Y. Tawk, S. E. Barbin and C. G. Christodoulou. Reconfigurable Antennas: Design and Applications. Proceedings of the IEEE, 103(3): 424-437, 2015.

M. Allayiti and J. R. Kelly. Multiple Parameter Reconfigurable Microstrip Patch Antenna. IEEE International Symposium, San Diego. 2017. doi:10.1109/apusncursinrsm.2017.8072613

M. Bataineh and T. Marler. Neural Networks. Elsevier. 1-9. 2017.

M. H. Beale, M. T. Hagan and H. B. Demuth. MatLab Neural Network Toolbox User's Guide, Natick MA, USA, 2013. Available via http://www.mathworks.com/help/nnet/ref/traingdm.html

M. Simsek, Q. Zhang, H. Kabir and N. Sengor. The recent Developments in Knowledge Based Neural Modelling. Elsevier-Science Direct, 1 (1):1321-1330, 2010.

Q. J. Zhang and K. C. Gupta. Neural networks for RF and microwave design. Artech House. 2000.

Z. Jiajie, W. Anguo and W. Peng. A survey on Reconfigurable Antennas. IEEE ICMMT Proceedings. 2008. 